\title{
Separating Rigid Motion for Continuous Shape Evolution
}

\author{
Niels Chr. Overgaard and Jan Erik Solem \\ Applied Mathematics Group, School of Technology and Society, Malmö University, Sweden
}

Received 2 October 2006; Accepted 14 July 2007

\begin{abstract}
A method is proposed for the construction of descent directions for the minimization of energy functionals defined for plane curves. The method is potentially useful in a number of image analysis problems, such as image registration and shape warping, where the standard gradient descent curve evolutions are not always feasible. The descent direction is constructed by taking a weighted average of the three components of the gradient corresponding to translation, rotation, and deformation. Our approach differs from previous work in the field by the use of implicit representation of curves and the notion of normal velocity of a curve evolution. Thus our theory is morphological and well suited for implementation in the level set framework.
\end{abstract}

Key Words: Computer Vision, Variational Problems, Gradient Descent, $L^{2}$-gradient, Level Set Methods, Normal Velocity, Rigid Motion, Shape Warping, Registration.

\section{Introduction}

Gradient descent curve evolutions occur frequently in image analysis applications. One popular example is the geodesic active contours [2]. Geodesic active contours is an example of shape optimization where curves are evolved to fit some form of data such as, for instance, image edges. Other examples are shape analysis applications such as shape warping and shape statistics. Shape statistics is often used as prior information in e.g. segmentation, cf. [10, 6].

Traditionally, shape analysis has been performed by studying the variation of landmarks on the curves, cf. e.g. [5]. The drawback of this approach is that landmarks are often very hard to find automatically. Performing analysis directly on the continuous curve overcomes this problem, but then registration of the shapes becomes much harder. Here we propose a method that has the potential of solving this registration problem. Also, a correct warping between shapes has the potential of solving the difficult "landmark correspondence" problem. In Section 4 we successfully apply the proposed method to both these problems.

In this paper we introduce a geometric procedure for decomposing any curve evolution into translation, rotation and deformation. This is useful for many applications and gives a way of modifying gradient flows. The decomposition is achieved by introducing orthogonal projections of the normal velocity of the evolution onto the subspaces generated by translations and rotations. Our investigation is inspired by the work in [4], where this type of decompositions were first studied. However, our method differs from theirs in that we use normal velocities which gives a geometric theory well suited for level set implementation, whereas [4] use

Correspondence to: nco@ts.mah.se

Recommended for acceptance by Dr. Sporring

ELCVIA ISSN:1577-5097

Published by Computer Vision Center / Universitat Autònoma de Barcelona, Barcelona, Spain 
vector-valued velocities allowing for tangential re-parametrization. This may seem like a small difference, but it turns out that the actual projections used are very different. We also show that the projected evolution still gives descent directions for the energy functional. It should be noted that similar questions have already been considered for deformable models working with elastic energy expressions for parametrized curves, surfaces, and bodies, see e.g., [12]. However, here we focus on methods that can be incorporated into the level set framework.

The present paper is an extended version of an earlier work [8] which has been presented at the 18th International Conference on Pattern Recognition in Hong Kong, August 2006.

\section{Level Sets, Normal Velocity, and $L^{2}$-Gradient Descent}

A simple closed curve $\Gamma$ can be represented as the zero level set of a function $\phi: \mathbf{R}^{2} \rightarrow \mathbf{R}$ as

$$
\Gamma=\left\{\mathbf{x} \in \mathbf{R}^{2} ; \phi(\mathbf{x})=0\right\} .
$$

The sets $\Omega^{\text {int }}=\{\mathbf{x} ; \phi(\mathbf{x})<0\}$ and $\Omega^{\text {ext }}=\{\mathbf{x} ; \phi(\mathbf{x})>0\}$ are called the interior and the exterior of $\Gamma$, respectively. Geometric quantities such as the outward unit normal $\mathbf{n}$ and the curvature $\kappa$ can be expressed in terms of $\phi$ as

$$
\mathbf{n}=\frac{\nabla \phi}{|\nabla \phi|} \quad \text { and } \quad \kappa=\nabla \cdot \frac{\nabla \phi}{|\nabla \phi|} .
$$

The function $\phi$ is usually called the level set function for $\Gamma$, cf. e.g. [7].

A curve evolution, that is, a time dependent curve $t \mapsto \Gamma(t)$, can be represented by a time dependent level set function $\phi: \mathbf{R}^{2} \times \mathbf{R} \rightarrow \mathbf{R}$ as $\Gamma(t)=\left\{\mathbf{x} \in \mathbf{R}^{2} ; \phi(\mathbf{x}, t)=0\right\}$. Let us consider the kinematics of curve evolutions. In the implicit representation, it does not make sense to "track" points on an evolving curve, as there is no way of knowing the tangential motion of points on $\Gamma(t)$. The important notion is instead that of normal velocity. The normal velocity of a curve evolution $t \mapsto \Gamma(t)$ is the scalar function defined by

$$
v(\Gamma)=\frac{d}{d t} \Gamma(t):=-\frac{\partial \phi(\mathbf{x}, t) / \partial t}{|\nabla \phi(\mathbf{x}, t)|} \quad(\mathbf{x} \in \Gamma(t)) .
$$

The normal velocity is independent of the curve representation (and the choice of level set function $\phi$ ) and is therefore a geometric quantity of the evolution. The set of normal velocities at $\Gamma$ is a linear space. It can be endowed with a natural scalar product and a corresponding norm, cf. [11],

$$
\langle v, w\rangle_{\Gamma}=\int_{\Gamma} v(\mathbf{x}) w(\mathbf{x}) d \sigma \quad \text { and } \quad\|v\|_{\Gamma}^{2}=\langle v, v\rangle_{\Gamma}
$$

where $v, w$ are normal velocities and $d \sigma$ is the curve length element. In the following we therefore denote the linear space of normal velocities at $\Gamma$ by $L^{2}(\Gamma)$.

The scalar product (4) is important in the construction of gradient descent flows for functionals $E(\Gamma)$ defined on a "manifold" $M$ of admissible curves $\Gamma$. Let the Gâteaux derivative of $E(\Gamma)$ at $\Gamma$ is denoted by $d E(\Gamma) v$, for any normal velocity $v$, and suppose that there exists a vector $\nabla E(\Gamma) \in L^{2}(\Gamma)$ such that

$$
d E(\Gamma) v=\langle\nabla E(\Gamma), v\rangle_{\Gamma} \quad \text { for all } v \in L^{2}(\Gamma) .
$$

Then $\nabla E(\Gamma)$ is called the $L^{2}$-gradient of $E$ at $\Gamma$. We make two remarks concerning this notion. First of all, not every functional $E=E(\Gamma)$ has an $L^{2}$-gradient, not even when the Gâteaux derivative exists. A concrete example is the Kimmel-Bruckstein functional, $E(\Gamma)=\int_{\Gamma}|\mathbf{w} \cdot \mathbf{n}| d \sigma$, for the optimal alignment of a curve $\Gamma$ to a given vector field $\mathbf{w}=\mathbf{w}(\mathbf{x}): \mathbf{R}^{2} \rightarrow \mathbf{R}^{2}$. It was shown in [9] that this functional has a well-defined Gâteaux derivative, however this derivative contains terms with Dirac $\delta$ 's which cannot be expressed using the scalar product defined in (4). Secondly, if the $L^{2}$-gradient does exist, then it is uniquely determined. This is essentially 
a consequence of the fact that any smooth function $v: \Gamma \rightarrow \mathbf{R}$ may be considered to be the normal velocity of some curve evolution which passes through $\Gamma$ at $t=0$, see [11, Lemma 2], so that $C^{\infty}(\Gamma)$ is dense in $L^{2}(\Gamma)$. Then, if $\tilde{\nabla} E(\Gamma)$ is "another" gradient for $E$ at $\Gamma$, then $\langle\tilde{\nabla} E-\nabla E, v\rangle_{\Gamma}=0$ for all normal velocities, by the definition of the gradient in (5), hence for all $v \in L^{2}(\Gamma)$ by density. In particular, we can take $v=\tilde{\nabla} E-\nabla E$ so that $\|\tilde{\nabla} E-\nabla E\|_{\Gamma}^{2}=0$, which proves the uniqueness assertion.

The gradient descent flow for the problem of minimizing $E(\Gamma)$ is defined as the solution of the following initial value problem

$$
\frac{d}{d t} \Gamma(t)=-\nabla E(\Gamma(t)), \quad \Gamma(0)=\Gamma_{0},
$$

where $\Gamma_{0}$ is an initial contour specified by the user.

Let us mention that in [4] the kinematic entity corresponding to our normal velocity $v$ in (3) is a vector valued function $\mathbf{v}: \Gamma \rightarrow \mathbf{R}^{2}$ given by $\mathbf{v}=v \mathbf{n}$. Consequently the $L^{2}$-scalar product used there is defined, via the Euclidean scalar product in $\mathbf{R}^{2}$, as $(\mathbf{v}, \mathbf{w})_{\Gamma}=\int_{\Gamma} \mathbf{v}^{T} \mathbf{w} d \sigma$. While $\langle v, w\rangle_{\Gamma}=(\mathbf{v}, \mathbf{w})_{\Gamma}$, for any pair of normal velocities, the difference in choice of scalar products actually makes a difference when rigid motions are considered, as we shall in the following sections.

\section{Decomposition of Evolutions}

Let $E(\Gamma)$ be an energy functional defined on the manifold $M$ of admissible curves. Again, we want to minimize $E(\Gamma)$. Instead of using the gradient descent evolution defined by (6), we search along the path of another evolution $t \mapsto \Gamma(t)$ defined by

$$
\frac{d}{d t} \Gamma(t)=v(\Gamma(t)), \quad \Gamma(0)=\Gamma_{0},
$$

where the normal velocity $v=v(\Gamma)$ is a descent direction for $E(\Gamma)$. The construction of $v(\Gamma)$ is based on an idea presented in [4]. The $L^{2}$-gradient $\nabla E=\nabla E(\Gamma)$, is decomposed into three components $\Pi_{T} \nabla E$, $\Pi_{R} \nabla E$, and $\Pi_{D} \nabla E$. Here $\Pi_{T} \nabla E$ and $\Pi_{R} \nabla E$ are the orthogonal projections of $\nabla E$ onto the subspaces of normal velocities at $\Gamma$ generated by translations and rotations, respectively. $\Pi_{D} \nabla E$ is defined as the residual $\Pi_{D} \nabla E=\nabla E-\Pi_{T} \nabla E-\Pi_{R} \nabla E$. The right-hand side in (7) is defined as a convex combination of these components,

$$
v=-\left(\mu_{1} \Pi_{T} \nabla E+\mu_{2} \Pi_{R} \nabla E+\mu_{3} \Pi_{D} \nabla E\right),
$$

where the weights $\mu_{1}, \mu_{2}, \mu_{3} \geq 0$ satisfy $\mu_{1}+\mu_{2}+\mu_{3}=1$.

Note that if we choose $\mu_{3}=0$ in (8), then the curve evolution (7) becomes a rigid motion; it changes the position and orientation of the initial contour $\Gamma_{0}$ without changing its shape. Hence the residual component $\Pi_{D} \nabla E$ may be interpreted as the part of $\nabla E$ responsible for the deformation of the contour shape. Also, note that if $\mu_{1}=\mu_{2}=\mu_{3}=1 / 3$, then $v=-\frac{1}{3} \nabla E$, so, apart from a time scaling, we recover the original gradient descent evolution (6).

\subsection{The Projection onto Translations}

We now show how the projections $\Pi_{T}$ is constructed. Let $\Gamma$ be a fixed contour, $\mathbf{v} \in \mathbf{R}^{2}$ an arbitrary vector, and define a curve evolution $t \mapsto \Gamma(t)$ as the translation of $\Gamma$,

$$
\Gamma(t)=\{\mathbf{x}+t \mathbf{v} ; \mathbf{x} \in \Gamma\} .
$$

It is easy to see that the normal velocity of the evolution in (9) is given by

$$
v_{T}=\mathbf{n}^{T} \mathbf{v}
$$

Inspired by this we define the following subspace of $L^{2}(\Gamma)$ :

$$
L_{T}=L_{T}(\Gamma):=\left\{v \in L^{2}(\Gamma) ; v=\mathbf{n}^{T} \mathbf{v} \text { for some } \mathbf{v} \in \mathbf{R}^{2}\right\} .
$$


The elements of $L_{T}$ are exactly the the normal velocities which come from pure translation motions. Notice that $\operatorname{dim} L_{T}=2$, because $L_{T}$ has the normal velocities $v_{1}=\mathbf{n}^{T} \mathbf{v}_{1}, v_{2}=\mathbf{n}^{T} \mathbf{v}_{2}$ as a basis, whenever $\mathbf{v}_{1}, \mathbf{v}_{2}$ is a basis for $\mathbf{R}^{2}$. Now, define $\Pi_{T}=\Pi_{T}(\Gamma)$ as the orthogonal projection in $L^{2}(\Gamma)$ onto $L_{T}$. Clearly, the identity

$$
\Pi_{T} v_{T}=v_{T}
$$

holds because $v_{T}$, given by (10), belongs to $L_{T}$. We can use this identity to find an explicit formula for $\Pi_{T}$. Multiply $v_{T}$ by $\mathbf{n}$ and integrate over $\Gamma$, then (10) implies that

$$
\int_{\Gamma} v_{T} \mathbf{n} d \sigma=\int_{\Gamma}\left(\mathbf{n}^{T} \mathbf{v}\right) \mathbf{n} d \sigma=\left[\int_{\Gamma} \mathbf{n n}^{T} d \sigma\right] \mathbf{v}
$$

We call the matrix $S:=\int_{\Gamma} \mathbf{n n}^{T} d \sigma$ appearing on the right-hand side the structure tensor for the curve $\Gamma . S$ is clearly positive semi-definite;

$$
\mathbf{w}^{T} S \mathbf{w}=\int_{\Gamma} \mathbf{w}^{T} \mathbf{n n}^{T} \mathbf{w} d \sigma=\int_{\Gamma}\left(\mathbf{n}^{T} \mathbf{w}\right)^{2} d \sigma \geq 0
$$

for any $\mathbf{w} \in \mathbf{R}^{2}$. However, more is true:

Proposition 1 The structure tensor $S$ is positive definite, in particular $S$ is invertible.

Proof: Suppose $\mathbf{w}_{0}^{T} S \mathbf{w}_{0}=0$ for some $\mathbf{w}_{0} \in \mathbf{R}^{2}$, then it follows from (14) that $\int_{\Gamma}\left(\mathbf{n}^{T} \mathbf{w}_{0}\right)^{2} d \sigma=0$, so that $\mathbf{n}^{T} \mathbf{w}_{0}=0$ identically on $\Gamma$. This implies that $\mathbf{n}$ is constant along $\Gamma$, which is clearly impossible if $\Gamma$ is a closed curve. This contradiction shows that $S$ must be positive definite.

We remark that the above results is invalid for one-dimensional curves in three of more space dimensions. In fact, the above proof breaks down of we consider a planar curve in three dimensions and take $\mathbf{w}_{0}$ normal to the plane in question.

By the proposition and (13) the translation vector $\mathbf{v}$ corresponding to the normal velocity $v_{T}$ can be reconstructed: $\mathbf{v}=\left[\int_{\Gamma} \mathbf{n n}^{T} d \sigma\right]^{-1} \int_{\Gamma} v_{T} \mathbf{n} d \sigma$. Using (10) we then get

$$
v_{T}=\mathbf{n}^{T} \mathbf{v}=\mathbf{n}^{T}\left[\int_{\Gamma} \mathbf{n n}^{T} d \sigma\right]^{-1} \int_{\Gamma} v_{T} \mathbf{n} d \sigma .
$$

Comparing this identity to (12) suggests that $\Pi_{T}$ is given by

$$
\Pi_{T} v=\mathbf{n}^{T} \mathbf{v}=\mathbf{n}^{T}\left[\int_{\Gamma} \mathbf{n n}^{T} d \sigma\right]^{-1} \int_{\Gamma} v \mathbf{n} d \sigma,
$$

for all normal velocities $v \in L^{2}(\Gamma)$. This is indeed true, as it is easily checked that the operator $\Pi$ defined by the right hand-side of $(16)$ is self-adjoint $\left(\Pi^{*}=\Pi\right)$ and idempotent $\left(\Pi^{2}=\Pi\right)$, hence an orthogonal projection. Moreover, (15) shows that $L_{T}$ is contained in the range of $\Pi$, and since the dimension of $\Pi$ 's range is two, it follows that $\Pi=\Pi_{T}$ as claimed in (16).

\subsection{The Projection onto Rotations}

Next, we derive a formula for the projection $\Pi_{R}$. Consider rotations in the plane; the rotation of $\Gamma$ about a point $\mathbf{x}_{0} \in \mathbf{R}^{2}$ with angular velocity $\omega$ is given by

$$
\Gamma(t)=\left\{R(t)\left(\mathbf{x}-\mathbf{x}_{0}\right)+\mathbf{x}_{0}: \mathbf{x} \in \Gamma\right\},
$$

where $R(t)=\left[\begin{array}{cc}\cos (\omega t) & -\sin (\omega t) \\ \sin (\omega t) & \cos (\omega t)\end{array}\right]$. The corresponding normal velocity at $t=0$ is given by

$$
v_{R}=\omega \mathbf{n}^{T}\left(\hat{\mathbf{x}}-\hat{\mathbf{x}}_{0}\right) \quad(\mathbf{x} \in \Gamma)
$$


Here we have defined $\hat{\mathbf{x}}=\left[\begin{array}{cc}0 & -1 \\ 1 & 0\end{array}\right] \mathbf{x}=R^{\prime}(0) \mathbf{x}$. Now, set

$$
L_{R}=\left\{v \in L^{2}(\Gamma) ; v=\omega \mathbf{n}^{T}\left(\hat{\mathbf{x}}-\hat{\mathbf{x}}_{0}\right) \text { for some } \omega \in \mathbf{R}\right\} .
$$

Clearly $\operatorname{dim} L_{R}=1$ for any fixed $\mathbf{x}_{0}$. The orthogonal projection onto $L_{R}$ is given by the formula

$$
\Pi_{R} v=\frac{\mathbf{n}^{T}\left(\hat{\mathbf{x}}-\hat{\mathbf{x}}_{0}\right) \int_{\Gamma} v \mathbf{n}^{T}\left(\hat{\mathbf{x}}-\hat{\mathbf{x}}_{0}\right) d \sigma}{\int_{\Gamma}\left|\mathbf{n}^{T}\left(\hat{\mathbf{x}}-\hat{\mathbf{x}}_{0}\right)\right|^{2} d \sigma} .
$$

Again it is easy to check that $\Pi_{R}^{*}=\Pi_{R}$ and $\Pi_{R}^{2}=\Pi_{R}$.

The point $\mathbf{x}_{0}$ in (19) is chosen such that the two subspaces $L_{T}$ and $L_{R}$ are orthogonal, or equivalently, $\Pi_{T} \Pi_{R}=\Pi_{R} \Pi_{T}=0$. Using (16) and (19) it is easy to see that $\mathbf{x}_{0}$ must satisfy the following vector relation $\int_{\Gamma}\left[\mathbf{n}^{T}\left(\hat{\mathbf{x}}-\hat{\mathbf{x}}_{0}\right)\right] \mathbf{n} d \sigma=0$, hence

$$
\hat{\mathbf{x}}_{0}=\left[\int_{\Gamma} \mathbf{n n}^{T} d \sigma\right]^{-1} \int_{\Gamma}\left(\mathbf{n}^{T} \hat{\mathbf{x}}\right) \mathbf{n} d \sigma,
$$

where the structure tensor for $\Gamma$ appears again. Since $L_{T}$ and $L_{R}$ are now orthogonal, it follows that the residual $\Pi_{D}=I-\Pi_{T}-\Pi_{R}$ ( $I$ denoting the identity operator) is also an orthogonal projection. The range of $\Pi_{D}$ is interpreted as the space of normal velocities which are responsible for deformations of the initial contour.

We end this section with some two important observations. The first observation implies that the normal velocity constructed in (8) is in fact a descent direction for the functional $E(\Gamma)$.

Proposition 2 If $\Pi$ is an orthogonal projection in $L^{2}(\Gamma)$, and the normal velocity $v(\Gamma)=-\Pi \nabla E(\Gamma)$ is not identically zero on $\Gamma$. Then $v(\Gamma)$ is a descent direction for $E(\Gamma)$.

Proof: Let $t \mapsto \Gamma(t)$ be the curve evolution which solves (7) with $v(\Gamma)$ given by the formula in the proposition, then the claim follows from the following simple calculation:

$$
\begin{aligned}
\frac{d}{d t} E(\Gamma) & =\langle\nabla E(\Gamma), v(\Gamma)\rangle_{\Gamma} \\
& =\langle\nabla E(\Gamma),-\Pi \nabla E(\Gamma)\rangle_{\Gamma}=-\|\Pi \nabla E(\Gamma)\|_{\Gamma}^{2}<0
\end{aligned}
$$

where we have used that $\Pi^{2}=\Pi, \Pi^{*}=\Pi$, and $v(\Gamma) \neq 0$.

The second observation is related to the fact that the projection methods described above can be applied to any energy functional $E$ with a well-defined $L^{2}$-gradient $\nabla E$. For instance we may apply the method to the arc length- and enclosed area functionals:

$$
E_{\circ}(\Gamma):=\int_{\Gamma} d \sigma, \quad \text { and } \quad E_{\bullet}(\Gamma):=\int_{\Omega^{\mathrm{int}}} d x
$$

respectively. Since the values of $E_{\circ}(\Gamma)$ and $E_{\bullet}(\Gamma)$ are invariant under translation and rotation, we would not expect these functionals to generate any rigid motion at all. In other words we expect the orthogonal projections onto $L_{T}(\Gamma)$ and $L_{R}(\Gamma)$ of the $L^{2}$-gradients

$$
\nabla E_{\circ}(\Gamma)=\kappa, \quad \text { and } \quad \nabla E_{\bullet}(\Gamma)=1
$$

to be zero. This expectation is easily verified by substituting the above gradients into the formulas (16) and (19) for the projections $\Pi_{T}$ and $\Pi_{R}$, and use the basic identities $\int_{\Gamma} \kappa \mathbf{n} d s=0, \int_{\Gamma} \mathbf{n} d s=0$, and the definition (20) of the centre of rotation $\mathbf{x}_{0}$. 


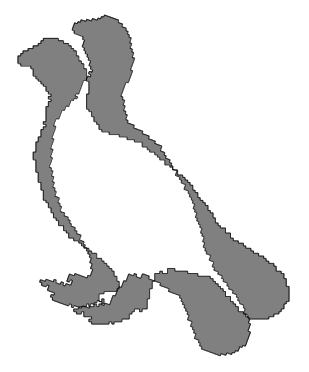

Figure 1: The figure shows the contours of two copies of the same pigeon. The symmetric difference of the interiors of these contours is the shaded region, i.e., the set of points belonging to exactly one of the interiors.

\section{Experiments}

In this section we apply the method of projections, introduced above, to some concrete examples. We consider two applications within shape analysis of curves: Continuous shape warping and registration of continuous shapes. All curves are represented implicitly as described in Section 2. The shapes are taken from the Kimia shape database [1].

We will use the the gradient flow associated with the area of symmetric difference, cf. [3], between two shapes $\Gamma=\left\{\mathbf{x} \in \mathbf{R}^{2}: \phi(\mathbf{x})=0\right\}$ and $\Gamma_{0}=\left\{\mathbf{x} \in \mathbf{R}^{2}: \phi_{0}(\mathbf{x})=0\right\}$ defined as

$$
E_{\mathrm{SD}}(\Gamma)=E_{\mathrm{SD}}\left(\Gamma, \Gamma_{0}\right)=\frac{1}{2} \text { area }\left(\Omega^{\text {int }} \triangle \Omega_{0}^{\text {int }}\right),
$$

where $A \triangle B$ denotes the symmetric difference of $A$ and $B$, defined as the set of points which is contained in exactly one of the sets $A$ of $B$, cf. Figure 1 . To find the gradient of the functional $E_{\mathrm{SD}}$, we introduce the characteristic functions $\chi_{\Omega^{\text {int }}}$ and $\chi_{\Omega_{0}^{\text {int }}}$ of the interiors of $\Gamma$ and $\Gamma_{0}$ respectively, and rewrite $E$ as,

$$
\begin{aligned}
E_{\mathrm{SD}}(\Gamma) & =\frac{1}{2} \int_{\mathbf{R}^{2}}\left(\chi_{\Omega^{\text {int }}}-\chi_{\Omega_{0}^{\text {int }}}\right)^{2} d \mathbf{x}=\frac{1}{2} \int_{\mathbf{R}^{2}}\left(\chi_{\Omega^{\text {int }}}^{2}-2 \chi_{\Omega^{\text {int }}} \chi_{\Omega_{0}^{\text {int }}}+\chi_{\Omega_{0}^{\text {int }}}^{2}\right) d \mathbf{x} \\
& =\frac{1}{2} \int_{\mathbf{R}^{2}}\left(\chi_{\Omega^{\text {int }}}-2 \chi_{\Omega^{\text {int }}} \chi_{\Omega_{0}^{\text {int }}}+\chi_{\Omega_{0}^{\text {int }}}\right) d \mathbf{x}=\int_{\Omega_{\text {int }}}\left(\frac{1}{2}-\chi_{\Omega_{0}^{\text {int }}}\right) d \mathbf{x}+\text { const }
\end{aligned}
$$

since the target contour $\Gamma_{0}$ is held fixed. It is now easy to see that the corresponding $L^{2}$-gradient is given by the normal velocity $\nabla E_{\mathrm{SD}}(\Gamma)=\frac{1}{2}-\chi_{\Omega_{0}^{\text {int }}}$ defined on $\Gamma$. In practice the characteristic functions are represented using continuous approximations of the Heaviside function, cf. e.g. [3].

\subsection{Continuous Shape Warping}

Here we show that the standard evolution from the symmetric difference gives a very un-intuitive motion when continuous shapes are warped from one shape to another. This has also been noted for the case of using approximate Hausdorff distance in [4]. If the shapes are not perfectly aligned, the evolution will remove details of the initial shape to a smooth shape and then grow new details corresponding to the target shape. This gives practically useless intermediate shapes. If we instead partition the flow as in (8) and weight rotation and translation higher than deformation, we obtain a much more intuitive flow with the desired intermediate shapes. We illustrate this in Figure 2. For each example the top row corresponds to the evolution where rigid motion projection is weighted higher than deformation and the bottom row is the unchanged symmetric difference flow.

\subsection{Registration of Continuous Shapes}

Another important application is shape registration. Shape registration implies the alignment of shapes and is a crucial step if one is interested in computing shape statistics and analyze shape variation. In this case we turn 

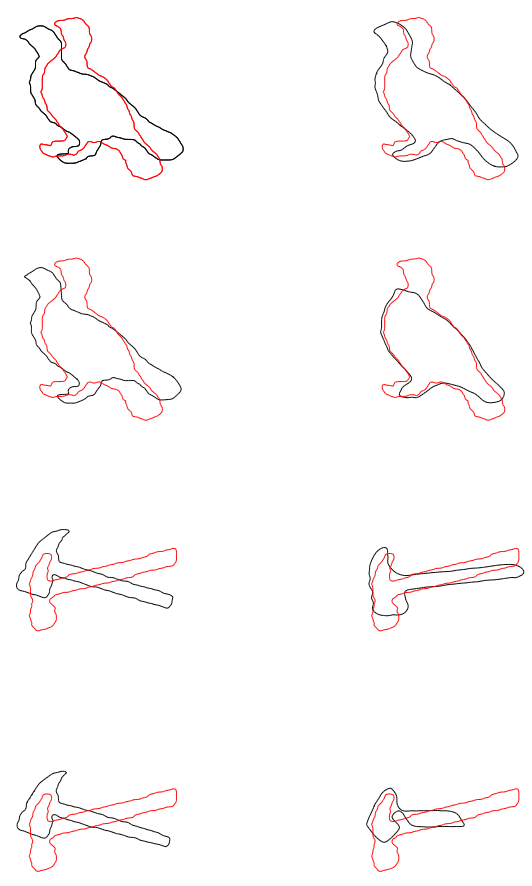
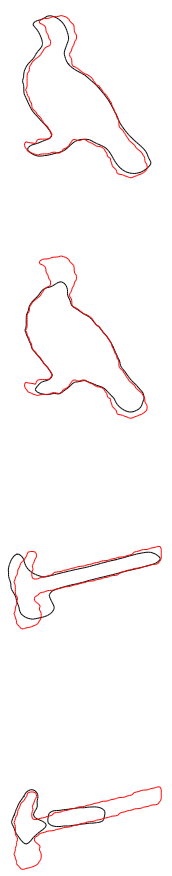
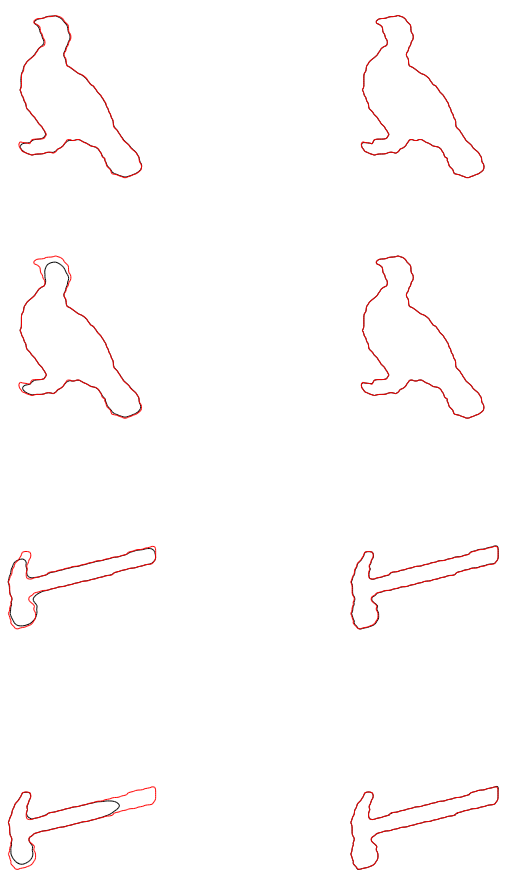

Figure 2: Examples of shape warping generated by minimizing the area of the symmetric difference between an evolving shape and the fixed target shape. The evolving shape is the black curve and the red curve is the target shape. The evolution is from left to right with the initial curve to the far left and the final curve to the far right. For each example, the top row corresponds to the evolution where the rigid motion projection is weighted higher than the deformation and the bottom row is the standard gradient descent flow. Notice that with the standard gradient descent flow, the intermediate shapes bear little or no resemblance to neither the initial nor the target shape. This problem can be solved using the weighted projected motion. The parameters used were $\left(\mu_{1}, \mu_{2}, \mu_{3}\right)=(0.3,0.7,0)$, initially, switching to $\left(\mu_{1}, \mu_{2}, \mu_{3}\right)=(0.1,0.1,0.8)$ at the end of the evolution.

off the deformation part entirely and simply use the normal velocity

$$
v(\Gamma)=-\left(\mu_{1} \Pi_{T} \nabla E_{\mathrm{SD}}(\Gamma)+\mu_{2} \Pi_{R} \nabla E_{\mathrm{SD}}(\Gamma)\right) .
$$

Figure 3 shows some examples of this procedure where one curve is chosen as the target shape and all other shapes are evolved towards this curve using (22).

\section{Conclusions}

We have presented a method for decomposing any curve evolution into rigid motion and deformation. The method is applied to shape warping and registration problems with satisfying results. The theory is developed for use in the level set framework and is simple to implement. It is our opinion that problems of shape analysis, shape statistics and shape optimization should be studied in the continuum framework using the language of geometry and mathematical analysis. Many vision problems can then be formulated as variational problems, which are usually easy to interpret, and discretizations are introduced only at the point where the numerical solution of the derived equations are computed. This will facilitate the understanding and comparison of different methods in the field. The aim of this paper was to try to apply level set methods to standard problems in shape analysis of curves. Although the method presented here is far from perfect, and certainly not competitive with 

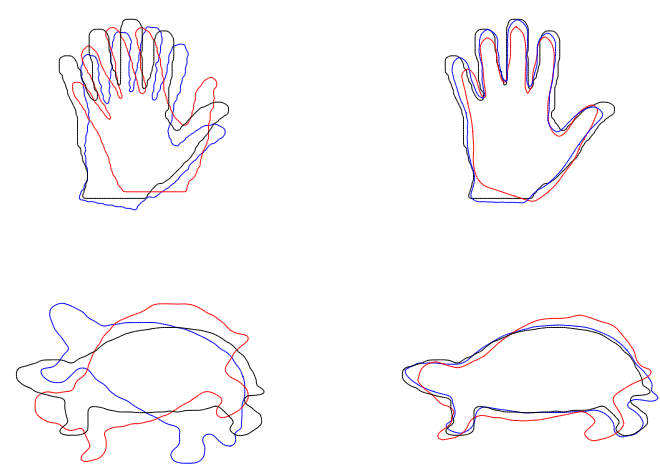

Figure 3: Registration using the rigid part of the evolution. The initial shapes (left), shapes registered (right).

standard tools in the field, it may still be regarded as a small step in the direction of a continuum formulation of shape analysis.

\section{References}

[1] The Kimia Shape Database. URL: http: //www. lems.brown.edu/vision/software/

[2] V. Caselles, R. Kimmel, and G. Sapiro. Geodesic active contours. Int. Journal of Computer Vision, 1997.

[3] T. F. Chan and W. Zhu. Level set based prior segmentation. Technical Report UCLA CAM Report 03-66, University of California at Los Angeles, 2003.

[4] G. Charpiat, R. Keriven, J-P. Pons, and O. Faugeras. Designing spatially coherent minimizing flows for variational problems based on active contours. In ICCV, Beijing, China, 2005.

[5] T. Cootes, C. Taylor, D. Cooper, and J. Graham. Active shape models - their training and application. Computer Vision and Image Understanding, 61(1):38-59, 1995.

[6] D. Cremers and S. Soatto. A pseudo-distance for shape priors in level set segmentation. In IEEE Workshop, Variational, Geometric and Level Set Methods in Computer Vision, 2003.

[7] S. J. Osher and R. P. Fedkiw. Level Set Methods and Dynamic Implicit Surfaces. Springer Verlag, 2002.

[8] N. Chr. Overgaard and J. E. Solem. Separating rigid motion for continuous shape evolution. In Proc. Int. Conf. on Pattern Recognition, Supplemental volume, pages 1-4, Hong Kong, 2006.

[9] N. Chr. Overgaard and J. E. Solem. An analysis of variational alignment of curves in images. In Scale Space 2005, LNCS 3459, pages 480-491, Springer-Verlag 2005.

[10] M. Rousson and N. Paragios. Shape priors for level set representations. In Proc. European Conf. on Computer Vision. Springer, 2002.

[11] J. E. Solem and N. Chr. Overgaard. A geometric formulation of gradient descent for variational problems with moving surfaces. In Scale Space 2005, LNCS 3459, pages 419-430, Springer-Verlag 2005.

[12] D. Terzopoulos and A. Witkin. Physically based models with rigid and deformable components. IEEE Comput, Graph. Appl., 8(6):41-51, 1998. 